\title{
A case of progressive subcortical gliosis presenting clinically as Steele-Richardson-Olszewski syndrome
}

\author{
R G WILL, A J LEES, W GIBB, R O BARNARD \\ From Maida Vale Hospital for Nervous Diseases, Maida Vale, London, UK
}

SUMMARY A patient presenting with the characteristic clinical features of Steele-RichardsonOlszewski syndrome is described, in whom neuropathological examination revealed atypical features, including extensive cortical and subcortical gliosis. The clinical and pathological features are discussed with particular reference to Creutzfeldt-Jacob disease and it is proposed that the case should be classified as progressive subcortical gliosis.

Progressive supranuclear palsy was recognised as a distinct clinical and pathological entity by Steele, Richardson and Olszewski in $1964,{ }^{1}$ and subsequent reports have confirmed their observations. ${ }^{2-4}$ In recent years, however, cases have been described with atypical features including pathologically confirmed cases without supranuclear ophthalmoplegia ${ }^{5}$ and typical clinical cases with unusual pathological features, including involvement of the neo-cortex. ${ }^{6} \mathrm{We}$ report a patient with the typical clinical and investigative features of Steele-Richardson-Olszewski syndrome in whom the pathological changes were distinct from this condition and were reminiscent of "progressive subcortical gliosis".

\section{Report}

A 58 year old mathematics teacher was admitted to the National Hospital for Nervous Diseases, Queen Square, in December 1978 for investigation of memory and gait disturbance. No history was available from the patient, but from her husband's account the patient had complained of difficulty focusing and reading print for 3 years. In the 6 months before presentation there had been a rapid deterioration in short term memory and for one month a disturbance of gait. This had progressed rapidly by the time of admission to hospital so that the patient required assistance to walk, with a tendency to fall backwards and to one side.

There was no significant past medical history and no family history of neurological disorder. The patient was not

Address for reprint requests: Dr R G Will, Maida Vale Hospital, London W9 1TL, UK.

Received 11 August 1987 and in revised form 15 March 1988. Accepted 2 April 1988 taking regular medication and had not in the past been exposed to neuroleptics. General physical examination was normal. The patient was disorientated in time and place and simple tests of arithmetic were performed briskly, but with numerous errors. The Babcock sentence was recalled at the third attempt and digit retention was six figures forwards and four backwards. The patient was, however, unable to retain any components of a name and address at 5 minutes and exhibited only patchy recall in simple testing of general knowledge. There was no dysphasia or dyspraxia, but there was a marked extra-pyramidal dysphonia. On neurological examination there was marked paucity of facial expression. Upward ocular pursuit movement was limited to $5^{\circ}$ and although horizontal and downward pursuit was full, all ocular movements were broken up. Voluntary saccadic upgaze was absent and downgaze impaired, while horizontal saccades were slow but of full range. the doll's head manoeuvre improved upgaze. There was no spontaneous nystagmus and optokinetic nystagmus was absent.

In the motor system there was marked axial rigidity with much milder rigidity of proximal limb musculature, associated with an intermittent coarse rest tremor of the right foot and hand. Muscle power was normal and tendon reflexes were brisk and symmetrical with flexor plantar responses. There was mild bilateral dysdiadochokinesis. Postural reflexes were impaired and the gait was broad-based and unsteady, such that the patient required assistance to walk.

Routine haematological and biochemical indices were normal and serology for syphilis was negative. Cerebrospinal fluid was acellular with a moderately elevated protein content of $80 \mathrm{mg} / 100 \mathrm{ml}$; cerebrospinal fluid protein electrophoresis did not reveal an oligoclonal pattern. An electroencephalogram was diffusely abnormal with generalised theta activity superimposed on a symmetrical and response background alpha rhythm. Enhanced CT brain scan was normal. Detailed psychological testing revealed that the patient was functioning within the dull average 

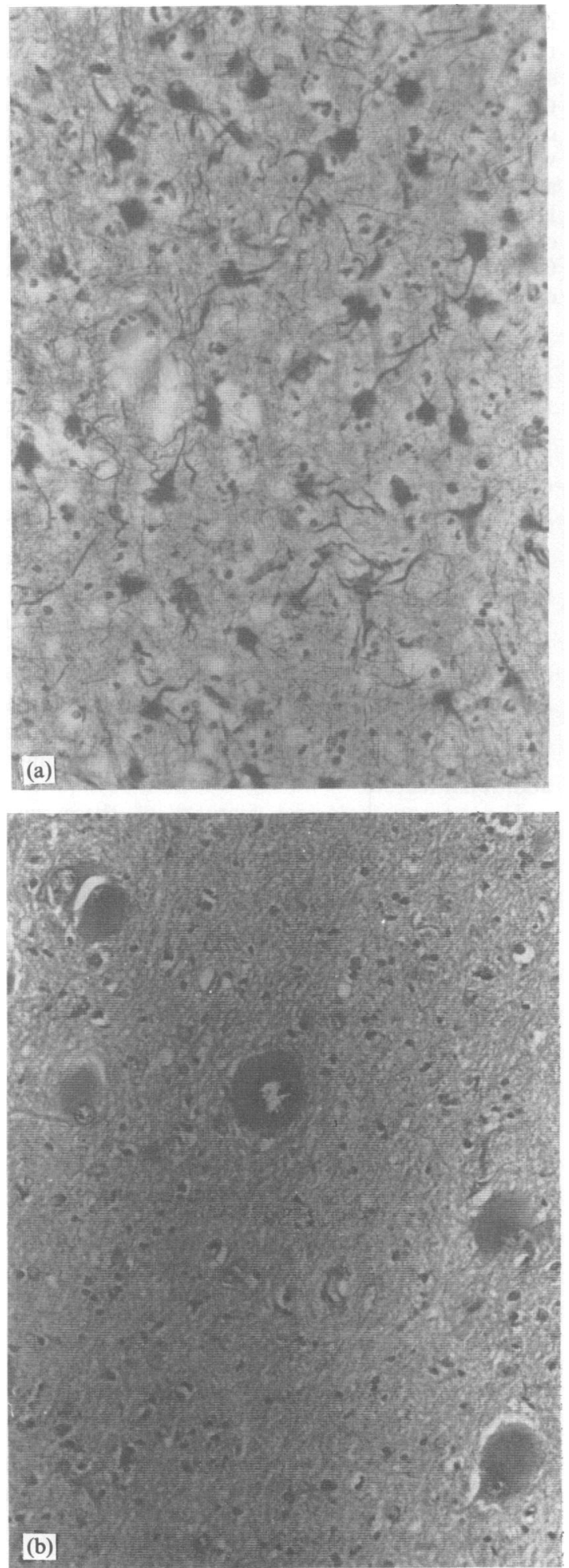

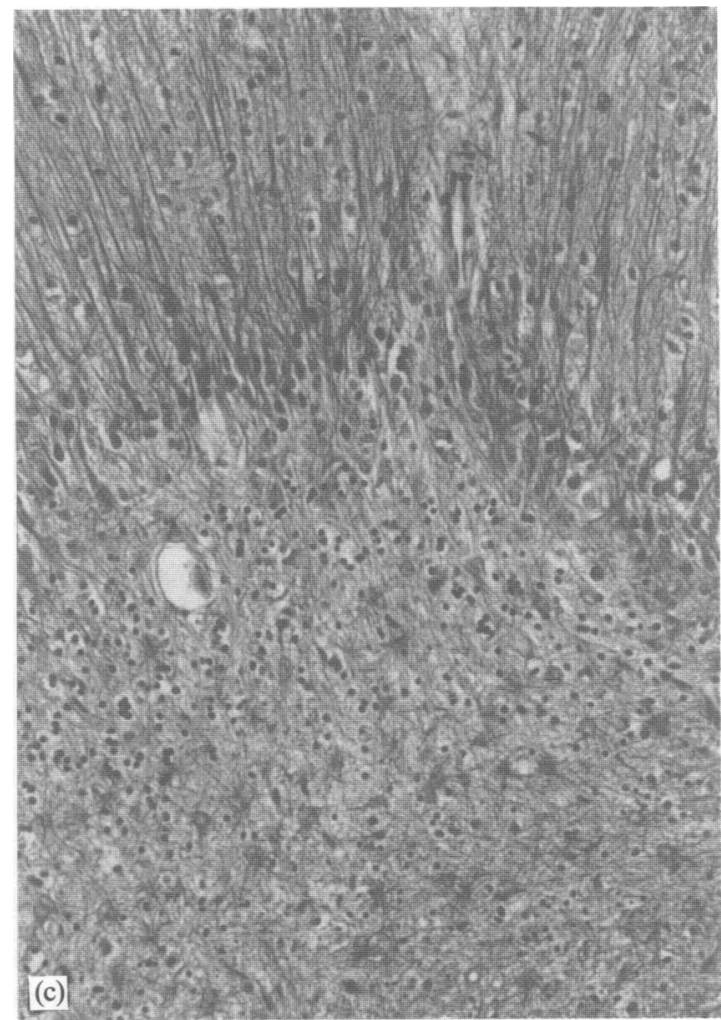

Fig (a) Frontal subcortical white matter. Astrocytic proliferation (PTAH $\times 375)$. (b) Abnormal giant

astrocytes $(H E \times 200)$. (c) Cerebellum. Severe isomorphic gliosis in the molecular layer; marked astrocytic proliferation in the granular layer $(P T A H \times 250)$.

range on non-verbal subtests, representing a moderate degree of general deterioration of intellectual function with poor memory. Neuro-otological examination confirmed the clinical findings of disordered conjugate gaze in the vertical plane and caloric testing revealed full downward gaze with cold stimulation and an improvement in upward gaze to $20^{\circ}$ on hot stimulation. The neuro-otological abnormalities were felt to be typical of a supranuclear ophthalmoplegia.

On the basis of the clinical features and the results of investigation a diagnosis of Steele-Richardson-Olszewski syndrome was made.

Following discharge there was a rapid deterioration in the patient's condition and by the time of admission to the local hospital on 31 January 1979 the patient was mute with no voluntary eye movement and marked axial rigidity. Over subsequent months the patient became unresponsive and doubly incontinent and developed a coarse rest tremor of the limbs. The patient died of bronchopneumonia on 5 July 1979 and necropsy was performed.

Pathology The brain weighed $1300 \mathrm{grm}$ and was stated to be soft in consistency.

Microscopy The meninges were unremarkable apart from slight generalised thickening. In the areas examined the cortical ribbon was of reasonable thickness and neuronal loss 
was patchy: no examples of neurofibrillary degeneration or argyrophile plaques were found. A striking feature was the extensive astrocytic proliferation in the cortex and subcortical white matter (fig, a). The astrocytes were chiefly of the gemistocytic type with bulky eosinophilic cytoplasm and oval vesicular nuclei: fibre-formation was common and a band of dense subpial gliosis was frequently present. Deep in the white matter occasional large astrocytes were present (fig, b), but the white matter myelin was normal: there were no signs of leucodystrophy. In Ammon's horn there was marked astrocytic proliferation but no other changes. In the central grey matter-caudate, putamen, globus pallidus-all appeared slightly shrunken and in all these nuclei there was marked astrocytosis with few normal neurons remaining. The internal capsule appeared normal, while the myelinated fibres within the globus pallidus were reduced. In the mamillary body neuronal fallout and astrocytosis were much less striking.

In the mesencephalon the periaqueductal grey matter and corpora quadrigemina were heavily involved with marked proliferation of large fibre-forming astrocytes. The astrocytosis did not involve the substantia nigra or the cerebral peduncles. In the upper pons astrocytosis was confined to the region around the fourth ventricle: the locus caeruleus, however, was normal. Large astrocytes were scattered amongst the neurons of nucleus pontis, but the fibre tracts were normal; in the medulla isolated large astrocytes were relatively few. The cerebellum showed severe atrophy and gliosis: the granule cells especially being reduced in number and replaced by hypertrophic astrocytes forming a semi-cystic meshwork of glial fibre (fig, c). Purkinje cells were reduced in number in a patchy distribution: in some folia less than one third remained. There was marked isomorphic gliosis of the molecular layer with fringes of fibres extending through the pia. The blood vessels were entirely normal. The astrocytes involved both in the cerebrum and cerebellum reacted strongly for glial fibrillary acidic protein.

\section{Discussion}

The clinical features in this patient, including supranuclear ophthalmoplegia of vertical gaze, dysarthria, axial rigidity and dementia, were characteristic of progressive supranuclear palsy. ${ }^{1-4}$ Investigation excluded a structural abnormality and neurootological examination confirmed a disorder of conjugate ocular movement typical of this syndrome. The neuropathological findings however were quite different from those previously described, ${ }^{1} 25$ with extensive diffuse astrocytosis of both cerebral cortex and subcortical regions with no evidence of neurofibrillary changes in the subthalamic region or brain stem. In the original description of Steele-RichardsonOlszewski syndrome ${ }^{1}$ gliosis was described as a characteristic and striking feature, but, in parallel to neuronal loss and neurofibrillary changes, was localised to the brain stem, diencephalon and cerebellum. In contrast there was no pathological evidence of frontal, cortical or white matter involvement. Subsequent studies ${ }^{125}$ have confirmed these character- istic histological features and, except for isolated reports of cortical neurofibrillary tangles, ${ }^{68}$ confirm sparing of the cerebral cortex and white matter from the pathological process. In the case reported here the cardinal pathological feature was intense gliosis, without neurofibrillary tangles, predominantly affecting the cortex and adjacent white matter. Despite the suggestive clinical features, this case cannot therefore be classified as Steele-RichardsonOlszewski syndrome and alternative pathological diagnoses must be considered.

In 1967 Neumann and $\mathrm{Cohn}^{7}$ described two patients with insidiously progressive dementia suggestive of Alzheimer's disease and two other patients with a clinical diagnosis of Pick's disease. The neuropathological findings in these four cases were similar and involved marked subcortical gliosis and astrocytic hyperplasia in the basal ganglia, thalamus, brain stem grey matter and ventral horns of the spinal cord. The cerebral cortex was normal with no evidence of neurofibrillary change, argyrophilic inclusions or swollen nerve cells. In a review of the literature 13 other cases were reported with a pathological appearance distinct from Alzheimer's disease and Pick's disease. A separate nosological entity, "progressive subcortical gliosis" was proposed, although it should be noted in the context of this report that in seven of the total of 13 cases cortical gliosis was a prominent additional feature.

The neuropathological features of these cases closely resemble the present case, both in the severity and distribution of the gliotic changes and the absence of the histological hallmarks of either Alzheimer's or Pick's disease. The clinical features differ considerably, however; in other reports of progressive subcortical gliosis $^{9-13}$ the commonest presentation is an isolated subcortical dementia, with no disorder of ocular movement. Only a minority of patients develop focal neurological signs, but these may include dysarthria, extrapyramidal rigidity, axial rigidity and a tendency to retropulsion. ${ }^{1214}$ One case of progressive subcortical gliosis has been described with oculomotor paresis as a prominent feature ${ }^{15}$ and this was of the supranuclear type (Seitelberger, personal communication), suggesting that in some patients with progressive subcortical gliosis the clinical features may be similar to progressive supranuclear ophthalmoplegia.

Gliosis, the cardinal pathological feature of the present case, is also a prominent pathological feature of transmissible spongiform encephalopathy ("Creutzfeldt-Jakob disease") and Shiraki and Mizutani ${ }^{16}$ have commented on the similarity of both the amyotrophic and thalamic [Stern-Garcin] variants of "Creutzfeldt-Jakob disease" to progressive subcortical gliosis of Neumann and Cohn. ${ }^{7}$ The failure to 
transmit either variant to primates ${ }^{17} 18$ suggests that such cases should be distinguished from transmissible subacute spongiform encephalopathy and supports the suggested nosological linkage with progressive subcortical gliosis. The characteristic clinical features of amyotrophic Creutzfeldt-Jakob disease (dementia, with Parkinsonism and wasting and fasciculation of limb musculature) differ from the clinical features in the present case and supranuclear ophthalmoplegia is not a feature of either of the above variants. Supranuclear ophthalmoplegia has however been described in familial cases of subacute, transmissible spongiform encephalopathy ${ }^{19-21}$ and it has been suggested that in "burnt out" cases of subacute CreutzfeldtJakob disease the typical spongiform change may be absent with gliosis as the major residual pathological feature. ${ }^{22}$ However, the absence of suggestive clinical features, including myoclonus and the characteristic encephalogram as well as the neuropathological findings, suggests that Creutzfeldt-Jakob disease is an unlikely diagnosis in the present case.

Other conditions associated with intense gliosis as a prominent neuropathological feature must also be considered. Multisystem atrophy, olivo-pontocerebellar atrophy and viral infections such as subacute sclerosing panencephalitis may all be associated with marked astrocytic proliferation, but the pathological features in this case exclude such diagnoses. Although the extensive astrocytic proliferation in this case is more suggestive of a reactive process the possibility of an extensive, infiltrative glioma has to be considered. Gliomas are frequently diffuse and in "gliomatosis" there may be widespread glial neoplasia in all parts of the nervous system. Gliomatosis, however, is often associated with developmental conditions such as neurofibromatosis or tuberose sclerosis ${ }^{23} 24$ and the microscopical appearances are usually those of pilocytic or fibrillary astrocytoma. The diagnosis of gliomatosis cannot be sustained on the pathology of the present case, and there are no clinical stigmata or family history suggesting "phakomatosis". Supranuclear palsy is not a feature of gliomatosis ${ }^{23}$ and it is of note that Neumann ${ }^{25}$ has described hyperplastic astrocytes in progressive subcortical gliosis.

The patient in this report exhibited clinical features characteristic of Steele-Richardson-Olszewski syndrome but neuropathological examination excluded this diagnosis. The disparity between the clinical and pathological features reinforces the importance of neuropathological confirmation of the diagnosis even in well established clinicopathological syndromes.

We thank Dr P C Gautier-Smith for permission to report this case. Professor W Blackwood carried out the post-mortem examination and kindly supplied the pathological material. We also thank Mrs Sheila Button for secretarial assistance.

\section{References}

1 Steele JC. Richardson JC, Olszewski J. Progressive supranuclear palsy. Arch Neurol 1964;10:333-59.

2 Behrman S, Carrole JD, Janota I, Matthews WB. Progressive supranuclear palsy: clinico-pathological study of four cases. Brain 1969;92:663-78.

3 Lees AJ. In: Fahn S, Marsden CD, eds. Movement Disorders, vol. 2. London: Butterworths, 1987;272-87.

4 Kristensen HO. Progressive supranuclear palsy-twenty years later. Acta Neurol Scand 1985;71:177-89.

5 Jellinger K, Riederer P, Tomonaga M. Progressive supranuclear palsy: clinico-pathological and biochemical studies. $J$ Neural Transm 1980; Suppl 16:111-28.

6 Ishimo H, Otsuki S. Frequency of Alzheimer's neurofibrillary tangles in the cerebral cortex in progressive supranuclear palsy (subcortical argyrophilic dystrophy). J Neurol Sci 1976;28: 309-16.

7 Neumann MA, Cohn R. Progressive subcortical gliosis. A rare form of presenile dementia. Brain 1967;40:405-18.

8 Mannen T, Toyokura Y, Tsukagoshi H, Miyatake T, Mohri N, Yokoyama T. Progressive supranuclear palsy: report of autopsied case. Adv Neurol Sci (Tokyo) 1972;16:497-503.

9 Hassin BB, Levitim D. Pick's disease-clinicopathological study and report of a case. Arch Neurol Psychiatry 1941;45:814-33.

10 Stern K, Reed E. Presenile dementia (Alzheimer's disease)-its pathogenesis and classification with two case reports. Am J Psychiatry 1945/46;102:191-7.

11 Minagawa M, Nakazato T. A special form of presenile dementia. Psychiat Neurol Jap 1973;75:79-93.

12 Masse G, Mikol J, Brion S. Atypical presenile dementia. J Neurol Sci 1981;52:245-67.

13 Moossy J, Martinez AJ, Hanin I, Rao G, Yonas H, Boller F. Thalamic and subcortical gliosis with dementia. Arch Neurol 1987;44:510-13.

14 Khoubesserian P, Davous P, Bianco C, et al. Demence familiale de type Neumann (gliose sous corticale). Rev Neurol (Paris) 1985;141:706-12.

15 Seitelberger F. Presenile gliale dystrophie. Acta Neuropathol 1968;:Suppl IV:109-18.

16 Shiraki H, Mizutani T. Simple poliodystrophic type of Creutzfeldt-Jakob disease. In: Mizutami T, Shiraki H, eds. Clinicopathological Aspects of Creutzfeldt-Jakob Disease. Amsterdam: Elsevier 1985:37-47.

17 Salazer AM, Masters CL, Gajdusek DC, Gibbs CJ. Syndromes of amyotrophic lateral sclerosis and dementia: relation to transmissible Creutzfeldt-Jakob disease. Ann Neurol 1983;14:17-26.

18 Little BW, Brown PW, Rodgers-Johnson P. Familial myoclonic epilepsy masquerading as Creutzfeldt-Jakob disease. Ann Neurol 1986;20:231-9.

19 Bertoni JM, Label LS, Sackelleres JC, Hicks SP. Supranuclear gaze palsy in familial Creutzfeldt-Jakob disease. Arch Neurol 1983;40:618-22.

20 Ross-Russell RWR. Supranuclear palsy of eyelid closure. Brain 1980;103:71-82.

21 Masters CL, Gajdusek DC. The spectrum of Creutzfeldt-Jakob disease and the virus-induced subacute spongiform encephalopathies. In: Smith WT, Cavanagh JB, eds. Recent Advances in Neuropathology, Vol. 2. Edinburgh: Churchill Livingstone, 1982:139-63.

22 Masters CL, Richardson EP. Subacute spongiform encephalopathy (Creutzfeldt-Jakob disease). The nature and progression of spongiform change. Brain 1978;101:333-44.

23 Grant N. Diffuse glioblastosis. In: Vinken PJ, Bruyn GW, eds. Handbook of Clinical Neurology. Amsterdam: N. Holland, 1975;18:73-79.

24 Sharenberg K, Jones E. Diffuse glioma of the brain in von Recklinghausen's disease: a study with silver carbonate. Neurology 1956;6:269-74.

25 Neumann MA. Pick's disease. J Neuropathol Exp Neurol 1949; 8:255-82. 